\title{
Desgaste profesional en los médicos de Atención Primaria de Barcelona
}

\author{
P. Sos Tena a , J. Sobrequés Soriano ${ }^{b}$, J. Segura Bernalc, E. Manzano Muletc, \\ C. Rodríguez Muñoz, M. García Garcíac, J. Cebriàb,c \\ Grupo Comunicación y Salud. a,b édicos de Familia y Psicólogos \\ ${ }^{a}$ CAP Rubí 1 Mutua de Terrassa. 'CAP San Miguel. Granollers. \\ Facultad de Psicología Blanquerna.URL. Barcelona
}

\section{RESUMEN}

Objetivo: describir el nivel de desgaste profe sional y estudiar su asociación con las caracterís ticas de los profesionales estudiados.

Diseño: estudio descriptivo transversal.

Emplazamiento: 93 centros de Atención Pri maria de la ciudad de Barcelona y de las comar cas del norte de la provincia: Vallés Oriental, Va llés Occidental, Maresme, Osona, Bages y Berguedà (región sanitaria centro).

Método: encuesta directa por entrevista perso nal o grupal a 528 médicos de Atención Primaria. Se administraron dos cuestionarios: uno de datos demográficos generales y el de desgaste profesio nal (Maslach Burnout Inventory-MBI).

Resultados: un 40\% de los encuestados presentaban algún sintoma de desgaste profesional sin diferencia entre sexos. De estos un 12,4\% mani festaban niveles muy altos de quemazón profesio nal.

Conclusiones: actualmente el porcentaje de médicos de Atención Primaria que muestran des gaste profesional es elevado, sobre todo los profe sionales de edad media (37-45 años), que trabajan más de 40 horas semanales y con más de 10 años en el mismo centro.

Palabras clave: Desgaste profesional. Atención Primaria. Médicos de familia.
Professional burnout in general practitioners in Barcelona

\section{ABSTRACT}

Objective: to describe job burnout and to analyse the influence of the sociodemographic va riables.

Design: observational descriptive transversal study.

Emplacement: 93 Primary Health Care centres in the province of Barcelona.

Method: individual or collective direct inter view to 528 Primary Health Care physicians. Two questioners were administrated for sociodemo graphic data and the Maslach Burnout Inventory (MBI).

Results: the $40 \%$ of the interviewed physicians had some burnout symptom without any difference between sexes. The $12.4 \%$ of these ones showed very high burnout levels.

Conclusions: nowadays the percentage gives in general practitioners show high burnout, mainly the professionals give half age (37-45 years old) that work more then 40 weekly hours than 10 years in the same center.

Key words: Burnout professional. Primary Health Care. Family physicians. 


\section{INTRODUCCIÓN}

El término desgaste profesional (burnout) se utiliza para describir un tipo de estrés laboral e institucional que se da específicamente en aquellas profesiones que mantienen una relación constante y directa con las personas que son los beneficiarios de su propio trabajo, en concreto personal sanitario, docentes, fuerzas de orden público, etc ${ }^{1}$. Este concepto fue utilizado en psicología por primera vez por Freudenberger (1974), aunque hoy en día no hay un consenso global para la explicación de este síndrome. Una de las teorías con más aceptación es la descrita por Maslach en la que el desgaste profesional estaría explicado por tres dimensiones interrelacionadas': cansancio emocional (CE), despersonalización (DP) y realización personal (RP).

Parece haber acuerdo en que la secuencia de síntomas de burnout es, en primer lugar, la ansiedad, pérdida del sentido del humor para posteriormente dar lugar a cambios cualitativos evidentes como el humor sarcástico y cinismo 3 .

Los primeros sentimientos negativos que se detectan son contra los desencadenantes del proceso, jefes y usuarios, después son los amigos y la familia y finalmente contra la propia persona, lo que supone una pérdida de autoestima y depresión al final del proceso. Otros síntomas asociados son los dolores de cabeza y molestias gastrointestinales, que pueden ir acompañados de pérdida de peso, insomnio y dificultad respiratoria ${ }^{4-6}$.

Las consecuencias para quien padece este síndrome pueden ser graves (desmotivación, frustración, depresión, drogodependencias), pero además trasciende a sus familiares (separaciones, malos tratos), a la empresa (absentismo, reducciones importantes del rendimiento, etc.) y, lo que es más grave si cabe, a sus pacientes con iatrogenia e insatisfacción en la relación médico-paciente ${ }^{7}$.

El objetivo principal de este artículo es el estudio del burnout en los médicos de Atención Primaria de Barcelona y la influencia de las variables sociodemográficas.

\section{MATERIAL Y MÉTODOS}

\section{Sujetos}

La población de estudio estaba formada por todos los profesionales que cumplían criterios de inclusión de 93 centros de Atención Primaria de la ciudad de Barcelona y de las comarcas del norte de la provincia: Barcelona ciudad, Maresme y Vallès Oriental, Vallès Occidental, Osona, Bages y Berguedà. Estos centros fueron seleccionados representando diferentes tipos existentes en la provincia de Barcelona y en base a una mejor organización de sus sistemas de información.

Los sujetos estudiados debían de cumplir los requisitos de ser médicos de Atención Primaria con mas de tres años de antigüedad y estar en activo en el momento del estudio. Se excluyeron odontólogos y pediatras. El total de médicos que cumplían estos criterios de los 93 centros participantes era de 737 .

\section{Material}

Los sujetos fueron invitados a contestar dos cuestionarios diferentes:

-Un cuestionario de datos generales que contenía preguntas sobre la situación personal y profesional de cada persona entrevistada.

Para caracterizar la situación laboral se preguntaba si el tipo de contrato laboral era fijo o interino, los años de experiencia profesional y los que había trabajado en el centro que ahora se encontraba ejerciendo la actividad laboral. También se exploró si ejerce como médico en otros centros, públicos o privados. Para describir el centro donde el médico desarrollaba su actividad principal se preguntaba si se trataba de titularidad publica (ICS) o privada, reformado (con dedicación de 6 horas diarias), y si estaba acreditado para la docencia de Medicina Familiar y Comunitaria (MFYC) . La última pregunta del cuestionario permitía al encuestado, si así lo deseaba, recibir el resultado de los cuestionaros de satisfacción y desgaste profesional.

\section{—El "Maslach Burnout Inventory (MBI)"15}

Este cuestionario fue validado por Maslach y Jackson apareciendo su versión definitiva en 1986. Se utilizó una versión traducida y validada de la original que ya ha sido utilizada en numerosos estudios realizados en el estado español.

Se trata de un cuestionario de 22 ítems con 7 opciones de respuesta (escala Likert de 0 a 6), que contiene las siguientes subescalas: cansancio emocional (CE): sentimiento del sujeto respecto a encontrarse saturado emocionalmente por el trabajo; despersonalización (DP): respuesta fría e impersonal hacia los pacientes; realización personal (RP): sentimientos de competencia y eficacia en la realización del trabajo.

Las puntuaciones de la escala total se obtienen al sumar los valores de los 22 ítems. Como puntos de corte se pensó en seguir los criterios seguidos por otros autores. Para ello se consideró que en la escala CE puntuaciones de 27 o superiores serían indicativos de un alto nivel de burnout, el intervalo entre 19 y 26 corresponderían a puntuaciones intermedias siendo las puntuaciones por debajo de 19 indicativas de niveles de "burnout" bajos o muy bajos. En la escala DP puntuaciones superiores a 10 serían nivel alto, de 6 a 9 medio, y menor 
de 6 bajo grado de despersonalización. En contrapartida, la escala RP funciona en sentido opuesto a las anteriores y así, de 0 a 33 puntos indicaría baja realización, de 34 a 39 intermedia y superior a 40 alta sensación de logro. Para el análisis de las escalas subescalas, se adaptaron los resultados a una escala de 0 a 100 mediante el porcentaje de puntos obtenidos sobre los posibles.

\section{Procedimiento}

Se consideró que el método de encuesta por correo podía producir un sesgo en la respuesta que desvirtuase los resultados, por lo que se optó por la encuesta directa por entrevista personal o grupal. Para ello se entrenó previamente a un equipo de psicólogos y estudiantes de último año de licenciatura de la facultad de Psicología Blanquerna de la Universidad Ramon Llull de Barcelona.

Durante el periodo comprendido entre enero y junio del año 2000 se acordó una cita con los directores de cada centro, normalmente en el horario de reunión de los médicos de los equipos de Atención Primaria. Los investigadores aprovecharon las reuniones para explicar el motivo del estudio y las instrucciones de rellenado de cada instrumento. En el caso de los centros no reformados se hicieron reuniones especiales donde la asistencia era optativa.
Los investigadores habían unificado los mensajes que debían dar a los encuestados y tenían instrucciones de no mencionar ciertas palabras (quemado, burnout, personalidad) que pudieran producir sesgos en la respuesta.

Se entregó un sobre en blanco con los dos cuestionarios: datos sociodemográficos generales y MBI. Todos los sujetos de manera individual y personalizada respondieron durante unos 45 minutos.

El estudio descriptivo incluyó las medidas de tendencia central, dispersión y de normalidad de la distribución de las variables sociodemográficas y dimensiones de los dos cuestionarios.

Para el análisis bivariante se han analizado todas la dimensiones del cuestionario MBI y las variables sociodemográficas (edad, género, estado civil, años de convivencia, trabajo de la pareja, número de hijos). Para las variables categóricas se analizó la varianza (ANOVA) y con las continuas la correlación de Pearson.

Los análisis estadísticos de los datos obtenidos se hicieron mediante el programa informático SPSS.

\section{RESULTADOS}

Obtuvimos respuesta de 586 médicos de Barcelona. El porcentaje de respuesta fue del $82 \%$. Un $57,9 \%$ de la población encuestada (Tabla I) son

\begin{tabular}{|c|c|c|c|}
\hline \multicolumn{4}{|c|}{ CARACTERISTICAS DESCRIPTIVAS DE LAS VARIABLES SOCIODEMOGRÁFICAS DE LOS MÉDICOS DE FAMILIA } \\
\hline & $\mathrm{n}$ & Media (DE) 0 inten & ntaje/ confianza $95 \%$ \\
\hline Edad & 582 & $42,7(7,7)$ & \\
\hline $28-36$ & & $19,2 \%$ & $26,46 \%-11,94 \%$ \\
\hline $37-45$ & & $47,5 \%$ & $66,33 \%-28,67 \%$ \\
\hline $46-55$ & & $24,4 \%$ & $31,36 \%-17,44 \%$ \\
\hline$\geq 56$ & & $8,9 \%$ & $12,65 \%-5,15 \%$ \\
\hline Sexo (\% hombres) & 582 & $58,3 \%$ & $58,49 \%-48,11 \%$ \\
\hline años de convivencia con la pareja & 488 & $14,8(8,4)$ & \\
\hline$\leq 5$ & & $27,4 \%$ & $34,22 \%-20,58 \%$ \\
\hline $6-10$ & & $18,1 \%$ & $25,36 \%-10,84 \%$ \\
\hline$\geq 11$ & & $54,5 \%$ & $59,9 \%-49,1 \%$ \\
\hline Número hijos que conviven familia & 578 & $1,3(1,1)$ & \\
\hline$<2$ & & $85,1 \%$ & $88,19 \%-82,01 \%$ \\
\hline$>2$ & & $14,9 \%$ & $18,52 \%-11,28 \%$ \\
\hline Viven en pareja & 508 & $85 \%$ & $88,1 \%-81,9 \%$ \\
\hline Tienen hijos & 448 & $76 \%$ & $79,95 \%-72,05 \%$ \\
\hline La pareja trabaja & 433 & $85 \%$ & $88,36 \%-81,64 \%$ \\
\hline
\end{tabular}


hombres frente a un $41,8 \%$ de mujeres. El $47,5 \%$ de médicos tiene entre $37-45$ años, y el $24,4 \%$ se encuentra entre las edades comprendidas entre los 46-55 años. El 84,9\% viven en pareja y un 54,5\% llevan conviviendo con la pareja más de 11 años, tienen hijos un $76 \%$ y la pareja trabaja en un $85 \%$ de los casos.

En la tabla II se muestran los resultados preguntados sobre los aspectos laborales. Los médicos encuestados trabajan en un ámbito de práctica urbana en un $70,6 \%$, suburbana un $12,5 \%$ y rural un 16,4\%. El 63,4\% de profesionales llevan más de 11 años trabajando en Atención Primaria, un 45,2\% están trabajando en el equipo de Atención Primaria actual menos de 5 años. Un 34,4\% de los médicos trabajan menos de 36 horas semanales, $37-46$ horas un 30,4\% y más de 47 un 35,1\%. Un $73 \%$ de los mismos tienen una situación laboral estable y un $39 \%$ trabaja en varios centros. Por lo que respecta al título de especialista un $65 \%$ responden positivamente y un $34 \%$ de los centros examinados ofrecen a la población atención continuada.

En el área estudiada un $91 \%$ pertenecen al Instituto Catalán de la Salud y están reformados un $90 \%$ de los centros. Un $28 \%$ funcionan como unidad docente y un $81 \%$ desean recibir información del cuestionario cumplimentado.

Las puntuaciones obtenidas en burnout y sus subescalas (cansancio emocional, despersonalización y falta de realización personal) para la población encuestada se expresan en la tabla III. La mediana de cansancio emocional es de 60, la de despersonalización de 50, y la de realización personal es de un 65. Un $40 \%$ de los profesionales estudiados presentan una puntuación alta en el MBI (Maslach Bur nout Inventory), siendo también entre un 40-45\% los que presentan puntuaciones altas en las tres escalas.

El análisis bivariado que se muestra en las tablas IV y V refleja que existe una asociación estadística

Tabla II

CARACTERÍSTICAS DE LOS PROFESIONALES Y DEL CENTRO DE SALUD

\begin{tabular}{|c|c|c|c|}
\hline & amaño de la muestra & Porcentajes y medidas & IC $95 \%$ \\
\hline Ámbito de práctica & 595 & & \\
\hline Urbana & & $70,6 \%$ & $66,25 \%-74,95 \%$ \\
\hline Suburbana & & $12,5 \%$ & $5,01 \%-19,98 \%$ \\
\hline Rural & & $16,4 \%$ & $9,07 \%-23,73 \%$ \\
\hline Años trabajados en APS & 547 & $14,1(7,8)$ & \\
\hline$\leq 5$ & & $11,5 \%$ & $19,02 \%-3,97 \%$ \\
\hline $6-10$ & & $25,1 \%$ & $32,03 \%-18,17 \%$ \\
\hline$\geq 11$ & & $63,4 \%$ & $65,87 \%-60,93 \%$ \\
\hline Años trabajados en el EAP & 558 & $7,2(6,2)$ & \\
\hline$\leq 5$ & & $45,2 \%$ & $51,13 \%-39,27 \%$ \\
\hline $6-10$ & & $31,1 \%$ & $37,75 \%-24,45 \%$ \\
\hline$\geq 11$ & & $23,7 \%$ & $30,7 \%-16,7 \%$ \\
\hline Horas trabajadas semanales & 598 & $46,4(16,6)$ & \\
\hline$<40$ & & $54,3 \%$ & $59,71 \%-48,89 \%$ \\
\hline$>40$ & & $45,7 \%$ & $51,6 \%-39,8 \%$ \\
\hline Situación laboral estable & 597 & $73 \%$ & $77,09 \%-68,91 \%$ \\
\hline Trabaja en varios centros & 593 & $39 \%$ & $45,3 \%-32,7 \%$ \\
\hline Tiene título de especialista & 593 & $65 \%$ & $69,77 \%-60,23 \%$ \\
\hline Tiene atención continuada el centro & 587 & $34 \%$ & $40,58 \%-27,42 \%$ \\
\hline Son del Instituto Catalán de la Salud (ICS) & 587 & $91 \%$ & $93,42 \%-88,58 \%$ \\
\hline Está reformado el centro & 593 & $90 \%$ & $92,54 \%-87,46 \%$ \\
\hline Funciona coma unidad docente el centro & 595 & $28 \%$ & $34,85 \%-21,15 \%$ \\
\hline
\end{tabular}


Tabla III

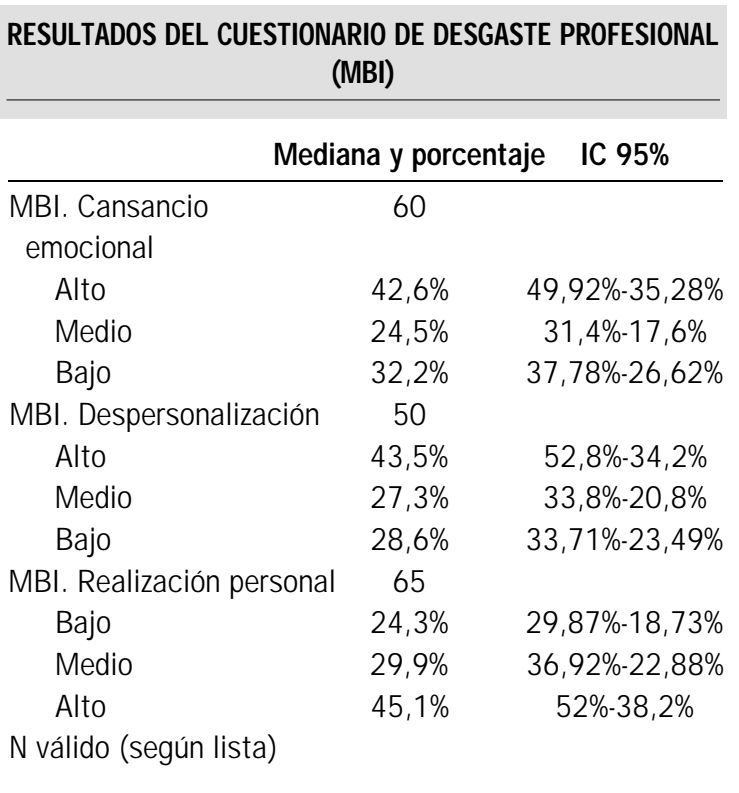

con el cansancio emocional y las edades comprendidas entre 37- 45 años que muestran puntuaciones más elevadas. La despersonalización se asocia con el trabajo profesional superior a las 40 horas semanales. Y la realización personal se asocia con tener más de 2 hijos.

\section{DISCUSIÓN}

Pensamos que el método de recogida de información utilizado es más útil que el de invitación por correo, ya que permite un nivel de respuesta mayor y más representativo de la población estudiada. Esto podría explicar en parte el alto índice de respuesta obtenido $(81 \%)$. Si bien no podemos conocer las características de las personas que no contestaron o no lo hicieron correctamente, el alto porcentaje de respuestas y la similitud de las variables sociodemográficas de los sujetos del estudio con los de la población general de médicos de Atención Primaria en nuestro medio, permite tener una seguridad razonable respecto a la validez de los resultados obtenidos ${ }^{8}$.

Los resultados de nuestro estudio están en la línea de los estudios publicados y las variables socio-demográficas estudiadas se correlacionaron de manera plausible con el desgaste profesional ${ }^{6,7,9,10}$.

En relación al sexo no hemos encontrado diferentes niveles de desgaste profesional entre hombres y mujeres. Aunque hay estudios que demuestran que el sexo femenino es un factor de riesgo para padecer desgaste profesional ${ }^{11,12}$, en general los estudios publicados hasta la fecha aportan resultados contradictorios ${ }^{13,14}$. Tradicionalmente se ha con- siderado que las mujeres pueden experimentar con más frecuencia múltiples fuentes de estrés, pero resultan consistentemente menos afectadas por el mismo (Greenglas, 1991) 5,15, las mujeres sobrellevan mejor que los hombres las situaciones conflictivas en el trabajo (Maslach y Jackson, 1985). Al margen de las diferencias biológicas en la susceptibilidad al estrés es obvio que existen diferentes roles que exponen a cada sexo a diferentes estresores y diferentes estrategias de afrontamiento. El principal estresor que perciben las mujeres, es el conflicto entre su cargo y su vida personal ${ }^{12}$. Las mujeres luchan por combinarlos, pero se aprecian mayor número de divorcios y menor número de hijos en comparación con los hombres.

La edad parece desempeñar un papel moderador, de manera que a más edad se evidenció un menor nivel de burnout, quizás porque el trabajador, según avanzan los años, adquiere una mayor seguridad en las tareas que desempeña y se hace menos vulnerable al estrés laboral. La experiencia de "quemarse" se da con mayor frecuencia en los jóvenes recién incorporados a su nuevo empleo. Estos sujetos no suelen estar debidamente preparados para hacer frente a los conflictos emocionales que desde el exterior se les presentan, y tienen menos experiencia en controlar sus propios sentimientos ${ }^{2,9}$. El perfil del profesional con agotamiento laboral, según nuestros resultados, es: varón próximo a los 37 años, casado, con 13 años de antigüedad en la profesión y con plaza en propiedad ${ }^{16}$.

En la literatura revisada la relación entre agotamiento profesional y los años de experiencia en el puesto de trabajo no aporta datos concluyentes. Tampoco existe evidencia de que el tiempo por sí mismo garantice la adquisición de estrategias de afrontamiento ni que las respuestas resulten apropiadas.

En un estudio británico, realizado en 1991 los profesionales más jóvenes y varones dieron resultados más altos en agotamiento emocional y despersonalización. La juventud también se encontró asociada a la desilusión de los médicos de Atención Primaria ${ }^{17}$. Si al estrés producido por el trabajo se le añade sobrecarga y poca valoración puede producir distrés, que se entiende como un el paso predecesor al burnout.

Las posibles explicaciones del distrés en jóvenes médicos son: a) dificultades para que los nuevos médicos desarrollen su ejercicio en un área metropolitana; b) algunos jóvenes médicos pueden tener expectativas no realistas sobre la naturaleza de la medicina general, y pueden sufrir reacciones emocionales en relación a los médicos más mayores que ajustaron sus expectativas; c) los más jóvenes estén más preparados para admitir las dificultades ${ }^{18}$; y d) prejuicios para valorar las cosas en general. 


\section{Tabla IV}

RELACIONES ENTRE EL DESGASTE PROFESIONAL Y LOS DATOS SOCIODEMOGRÁFICOS

\begin{tabular}{lccc}
\hline & Cansancio emocional & Despersonalización & Realización personal \\
\hline Edad & $50^{*}$ & 55 & 65 \\
$28-36$ & $65^{*}$ & 50 & 60 \\
$37-45$ & $55^{*}$ & 50 & 70 \\
$46-55$ & $55^{*}$ & 50 & 68 \\
$\quad>56$ & & & 65 \\
Sexo & 60 & 50 & 65 \\
Mujer & 60 & 50 & 60 \\
Hombre & & & 65 \\
Años de convivencia & 60 & 60 & 65 \\
$<5$ & 60 & 50 & $60 *$ \\
$6-10$ & 60 & 50 & $73^{*}$ \\
$>11$ & & & 65 \\
Número de hijos & 60 & 50 & 65 \\
$<2$ & 48 & 50 & 65 \\
$>2$ & 60 & 50 & 50 \\
Vive en pareja & 60 & 53 & \\
Tiene hijos & 60 & 50 pareja trabaja
\end{tabular}

Resultados pruebas estadísticas comparación de medias (ANOVA). $p<0,05 * p<0,001$

\section{Tabla V}

\section{RELACIONES ENTRE EL DESGASTE PROFESIONAL Y LOS DATOS PROFESIONALES Y DEL CENTRO DE SALUD}

\begin{tabular}{|c|c|c|c|}
\hline & Cansancio emocional & Despersonalización & Realización personal \\
\hline \multicolumn{4}{|l|}{ Ámbito de práctica } \\
\hline Urbana & 60 & 50 & 65 \\
\hline Suburbano & 65 & 50 & 65 \\
\hline Rural & 55 & 55 & 65 \\
\hline \multicolumn{4}{|l|}{ Años EAP } \\
\hline$<5$ & 58 & 60 & 60 \\
\hline $6-10$ & 60 & 50 & 65 \\
\hline$>10$ & 55 & 50 & 65 \\
\hline \multicolumn{4}{|l|}{ Horas de trabajo semanal } \\
\hline$<40$ & 60 & $50 *$ & 65 \\
\hline$>40$ & 60 & $55^{*}$ & 65 \\
\hline Situación laboral estable & 60 & 50 & 65 \\
\hline Trabaja en varios centros & 55 & 55 & 65 \\
\hline Tiene título especialista & 58 & 50 & 65 \\
\hline Centro con atención continuada & 60 & 55 & 65 \\
\hline Centro titularidad pública & 60 & 50 & 65 \\
\hline Centro reformado & 60 & 53 & 65 \\
\hline Centro acreditado docente & 55 & 50 & 65 \\
\hline Desea recibir información & 60 & 50 & 65 \\
\hline
\end{tabular}

Resultados pruebas estadísticas comparación de medias (ANOVA). $p<0,05 * p<0,001$ 
Según otros autores ${ }^{2,13}$ el desgaste profesional requiere un periodo de latencia para que actúen sus desencadenantes, los mayores de 55 años parecen un tanto inmunizados frente al síndrome, predominando en las edades medias de la vida laboral.

Respecto a las variables familiares, aparece que la paternidad se relaciona con un menor cansancio emocional fruto, a nuestro parecer, de un mejor afrontamiento de conflictos y estrés laboral ${ }^{2}$, aunque también sea razonable asumir cierto nivel de confusión de estos aspectos con la edad del profesional.

En resumen, los médicos de Atención Primaria de nuestra muestra manifiestan unos niveles de agotamiento profesional o de burnout elevado en un $40 \%$, siendo $12 \%$ de los resultados extremos, con los consiguientes perjuicios que conlleva para la salud individual y colectiva.

En los últimos años la visión humanista de la medicina ha chocado con el planteamiento de un sistema sanitario cada vez más deshumanizado y despersonalizado al que deben adaptarse los profesionales. Al mismo tiempo, la reforma de la Atención primaria y la escasez de recursos ha conlleva- do que la dedicación del profesional haya aumentado de forma considerable, y que las expectativas de su implicación emocional con los pacientes sea más intensa ${ }^{19}$. Se sabe que la complejidad de la práctica médica junto con los recientes cambios políticos, sociales y económicos de los médicos son las primordiales causas del incremento de estrés en los médicos ${ }^{16,20}$.

Estos datos sugieren la necesidad de profundizar en las causas de este fenómeno, de forma que se puedan diseñar mecanismos de intervención, que corrijan estas tendencias, y redunden en una mejora de la calidad asistencial y organizacional de las áreas básicas y de su eficiencia ${ }^{10}$.

\section{CORRESPONDENCIA:}

Pilar Sos Tena

CAP RUBI 1 (Mutua de Terrassa)

C/ Prat de la Riba 20-22

08191 Rubí (Barcelona)

Tel. $935886035 \quad$ Fax 935882037

e-mail: pilarsos@yahoo.es

\section{Bibliografía}

1. Pines A, Aronson E, Kafry D. Burnout: From tedium to personal growth. New York: Free Press. 1981.

2. Alvarez Gallego E, Fernández Ríos L. El Síndrome de Burnout o el desgaste profesional 1: revisión de estudios. Revista de la Asociación Española de Neuropsiquiatría 1991: 257-65.

3. Duch FR, Ruiz de Porras L, Gimeno D. Síndrome de burnout en Atención Primaria. Salud Rural 1999; 8: 41-52.

4. Freudenberger HJ. The staff burnout syndrome in alternative institutions. Psicotherapy theory research and practice $1975 ; 12$.

5. Torrado Oubiña V, Fernández Ríos L, Martínez Calvo C. Estrés laboral y estado de salud entre psicólogos clínicos y psiquiatras. Clínica y Salud 1992; 7: 163-80.

6. De la Fuente L, De la Fuente E. Burnout y satisfacción laboral: indicadores de salud laboral en el ámbito sanitario. Clínica y Salud 1997; 8: 481-94.

7. De Pablo González R, Suberviola González JF. Prevalencia del síndrome de burnout o desgaste profesional en los médicos de Atención Primaria. Aten Primaria 1998; 22 (9): 5804.

8. Cebrià J, Segura J, Corbella S, Sos P, Comas O, García M. Rasgos de personalidad y burnout en médicos de familia. Aten Primaria 2001; 27: 459-68.

9. Olivar Castrillón C, González Morán S, Martinez Suárez MM. Factores relacionados en la satisfacción laboral y el desgate profesional en los médicos de atención primaria de Asturias. Aten primaria 1999; 24: 352-9.

10. Bustinza Arriortua A, López-Herce Cid J, Carrillo Álvarez A, Vigil Escribno MD, De Lucas García N, Panadero Carlovilla E. Situación de burnout de los pediatras intensivistas españoles. Anales Españoles de Pediatría 2000; 51: 28-33.
11. Thomson S, Soares J, Nolan P, Dallender J, Arnetz B. Feeling of professional fulfilment and exhaustion in mental health personnel: The importance of organisational and individual factors. Psycother Psychosom 1999; 68: 157-64.

12. Byone G. Stress in women doctors. British Journal of Hospital Medicine 1994; 51: 267-8.

13. Arnetz B. Physicians'view of their work environment and organization. Psychotherapy Psychosom 1997; 66: 15562.

14. Richardsen AM, Burke RJ. Occupational stress and job satisfaction among physicians: sex differences. Soc Sci Med 1991; 33: 1179-87.

15. Maslach C, Jackson SE. The Maslach Burnout Inventory. 2nd ed. Palo Alto Consulting Psychologist Press, 1986.

16. Hidalgo Rodrigo I, Díaz González R. Estudio de la influencia de los factores laborales y sociodemográficos en el desarrollo del síndrome de agotamiento profesional en el área de medicina especializada del Insalud de Avila. Med Clínica 1994; 103: 408-12.

17. Winefield H, Anstey T. Job stress in General Practice: practitioner age, sex and attitudes as predictors. Family Practice 1991; 8: 140-4.

18. Carmel S, Glik S. Compassionate-empathic physicians: personality traits and social-organizational factors that enhance or inhibit this behavior pattern. Soc Sci Med 1996; 43: 1253-61.

19. De la Gándara Martín JJ, De Dios Francos A, De Diego Herrero E, Goñi Labat AI, Hernández Herrero H, Pozo de Centro JV. Estudio de la morbilidad psiquiátrica en personal sanitario. Actas Luso. Esp Neurol Psiquiatría 1994; 22: 71-6.

20. Orman M. Physician stress: is it inevitable? Missouri Medicine 1989; 86: 21-5. 